\title{
Physīcs
}

VIEWPOINT

\section{Structure Formation in the Very Early Universe}

\author{
Numerical calculations explain how density fluctuations in the Universe grew by orders of \\ magnitude during the "primordial dark ages."
}

\section{by Mark P. Hertzberg*}

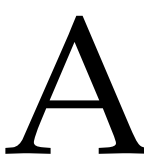

fundamental task of modern cosmology is to understand the formation of structure in our Universe from its earliest moments. The leading idea is that of inflation [1, 2], which can successfully explain many observed features of our Universe, from its flatness to its isotropy. Inflation produces an ex-

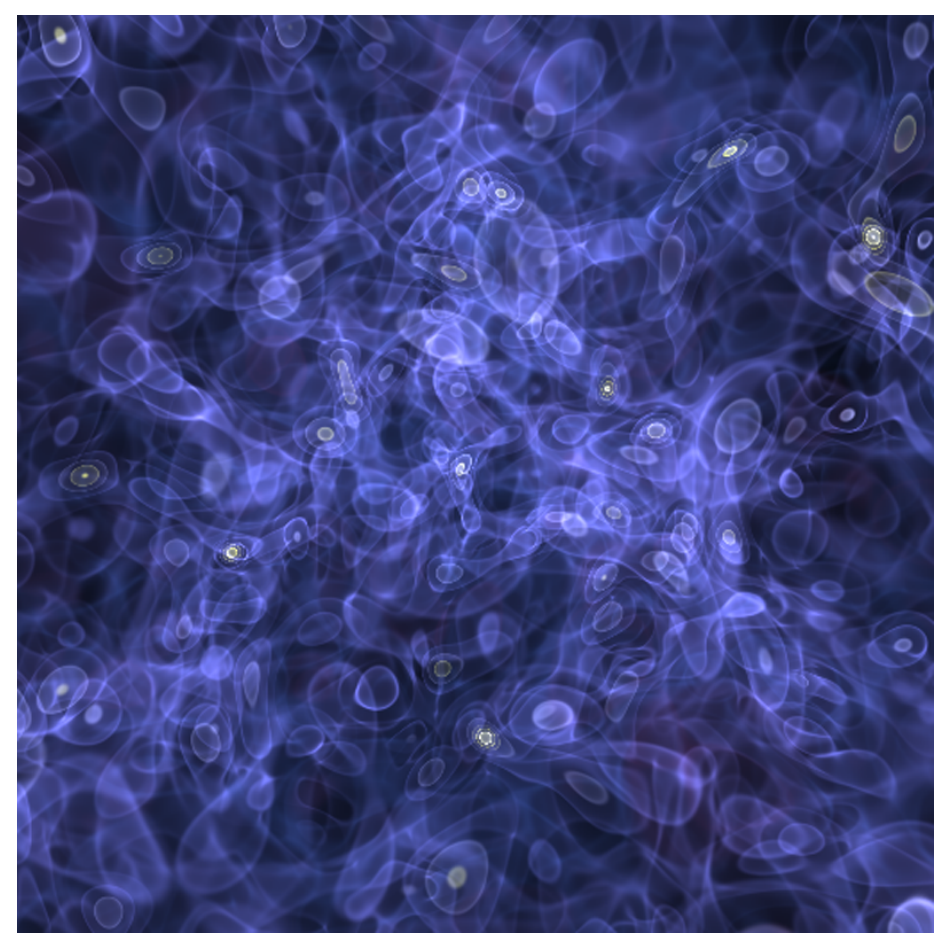

Figure 1: $3 \mathrm{D}$ rendering of density fluctuations calculated for a universe that has expanded 200 -fold since the end of inflation (brighter colors indicate larger densities). Musoke et al. find that small-amplitude fluctuations at the end of inflation are amplified by several orders of magnitude. (N. Musoke et al., Phys. Rev. Lett. (2020))

*Institute of Cosmology, Tufts University, Medford, MA, USA tremely homogeneous Universe, but quantum fluctuations create fluctuations in density from place to place. These fluctuations become the seeds of the large-scale structure of today's Universe- - a foam-like texture of voids and galaxy clusters, sheets, and filaments. However, it is hard to explain how the initial fluctuations grew in amplitude by many orders of magnitude, leading to the present density variations. Nathan Musoke and colleagues from the University of Auckland, New Zealand, take small initial quantum fluctuations and predict their evolution with a high-precision numerical computation [3]. By focusing only on a specific regime-very early times and very small spatial scales-they are able to track the evolution of these fluctuations within a self-consistent computation. They predict the development of complicated structures with density contrasts that are orders-of-magnitude larger than the initial fluctuations. The results shed light on structure formation in the first stages after inflation. They could also help researchers pinpoint new observational signatures of the earliest moments of the Universe.

The basic assumption of inflation is that the very early Universe was filled with bosonic particles in a degenerate quantum state. These spinless particles, known as inflatons, are the quanta of a primordial field carrying a large potential energy that drove an exponential expansion of the Universe. Researchers have shown that, if they include the inflaton field in Einstein's field equations, they can predict a phase of rapid exponential expansion, in which the Universe grew in size by over 30 orders of magnitude.

This inflationary expansion would lead to a universe teeming with inflaton particles distributed with extremely uniform density. Describing the evolution of the inflaton distribution during the exponentially expanding phase is moderately straightforward to do, at least for the simplest models for inflation. The inhomogeneities deriving from initial quantum fluctuations are very small-deviations in local density are of order of 1 part in 100,000. In this regime, the corresponding Einstein equations can be simplified to account only for linear perturbation terms-a readily tractable case.

However, understanding what happens right after inflation is much more difficult. In this postinflationary 
era-dubbed the primordial dark ages because of the absence of any photons - the inflaton dominates the Universe's energy density, but all sorts of nonlinear effects can quickly become important and amplify inhomogeneities. The inflatons can interact with each other, causing them to aggregate into spatially distinct patches. Inflatons can also decay into and couple to standard model particles. And gravitational interactions between all these various particles could become relevant [4].

To gain some insight into this era, Musoke et al. follow an approach explored by previous work [5-8], which focused on only one of those nonlinear factors: the interactions of inflatons among themselves. Numerical studies of this postinflationary era have recently been performed, but the current work goes a step further by improving the numerical code to track as accurately as possible the evolution from the linear to nonlinear regime.

Musoke et al. study the evolution of inflaton distribution by numerically solving with high precision the corresponding field equations coupled to gravity. This leads to a phase in which inflatons break up into a highly inhomogeneous configuration, and the system enters a nonrelativistic regime as the particles' wavelengths stretch and their momenta decrease. The researchers call this the matter-dominated phase, as its evolution mirrors that which would be observed with conventional matter. However, since the system is still highly degenerate, it cannot be described by classical particle physics but, instead, by classical field theory. To describe these conditions, Musoke et al. use the so-called Schrödinger-Poisson system of equations, which is known to be numerically tractable [9]. The team relied on a numerical code called PyUltraLight. Partly developed by them, the code allows for a more accurate numerical treatment of the Schrödinger-Poisson equations.

In the matter-dominated era, density fluctuations grow with time. This is because gravity is attractive, so overdense regions attract their surroundings and become even denser. Starting from very small initial fluctuations Musoke et al. observe that fluctuations get amplified by several orders of magnitude, reaching or exceeding unity on some spatial scales (Fig. 1). This regime is precisely where linear approximations fail. Thanks to their high-precision 3D numerical calculations, the team can tackle this regime and obtain statistical details of the resulting inflaton distribution.

The new work focuses only on inflatons and their selfinteractions and is thus suitable to describe a very early Universe dominated by such particles. The natural next step would be to include interactions of the inflaton with standard model particles and with other particles, including dark matter, into which inflatons should eventually decay. This inclusion would allow researchers to connect inflation with the later Universe made up of both baryonic matter and dark matter. Next, researchers will need to expand the temporal range of the calculations to describe the emergence of the large-scale structure that we see in the Universe today. Although these later regimes would be very different from the primordial dark ages, some of the computational techniques employed in this work could prove useful to the task.

Finally, it's important to determine whether there are any observable features that could allow researchers to probe this early postinflationary phase. Musoke et al. argue that such features exist. They suggest that the growth of early fluctuations may produce gravitational fields sufficiently strong to generate detectable gravitational waves. These waves would appear as a stochastic background, much like the cosmic microwave background. Depending on the energy scale of inflation, these gravitational waves might fall in a frequency range accessible to existing detectors like LIGO and Virgo or to future space-based detectors like the Laser Interferometer Space Antenna (LISA), planned for launch in 2034.

This research is published in Physical Review Letters.

\section{REFERENCES}

[1] A. H. Guth, "Inflationary universe: A possible solution to the horizon and flatness problems," Phys. Rev. D 23, 347 (1981).

[2] A.D. Linde, "A new inflationary universe scenario: A possible solution of the horizon, flatness, homogeneity, isotropy and primordial monopole problems," Phys. Lett. B 108, 389 (1982).

[3] N. Musoke et al., "Lighting the dark: Evolution of the postinflationary universe," Phys. Rev. Lett. 124, 061301 (2020).

[4] L. Kofman et al., "Towards the theory of reheating after inflation," Phys. Rev. D 56, 3258 (1997).

[5] G. N. Felder and I. Tkachev, "LATTICEEASY: A program for lattice simulations of scalar fields in an expanding universe," Comput. Phys. Commun. 178, 929 (2008).

[6] A. V. Frolov, "DEFROST: A new code for simulating preheating after inflation," J. Cosmol. Astropart. Phys. 2008, 009 (2008).

[7] M. A. Amin et al., "Oscillons after inflation," Phys. Rev. Lett. 108, 241302 (2012).

[8] K. D. Lozanov and M. A. Amin, "Equation of state and duration to radiation domination after inflation," Phys. Rev. Lett. 119, 061301 (2017).

[9] M. A. Amin and P. Mocz, "Formation, gravitational clustering, and interactions of nonrelativistic solitons in an expanding universe," Phys. Rev. D 100, 063507 (2019).

10.1103/Physics. 13.16 